\title{
Impact du nombre d'interventions infirmières sur la durée d'administration des catécholamines chez les patients de réanimation : étude INTERVAL
}

\author{
INTERVAL Impact of Nurses' Interventions on Vasopressors Weaning: the INTERVAL Study
}

\begin{abstract}
A. Youssoufa - G. Decormeille $\cdot$ P. Michel · G. Jacq · F. Brouard · N. Aissaoui · S. Barbar $\cdot$ N. Belaizi $\cdot$ F. Boissier $\cdot$ C. Boulinguiez $\cdot$ V. Chauvin $\cdot$ C. Corrolleur $\cdot$ S. Dame $\cdot$ D. Da Silva $\cdot$ J. Dauvergne $\cdot$ J. Domitien $\cdot$ G. Fouquet C. Garin · P. Gay · D. Grimaldi · O. Hamzaoui · A. Joosten · A. Kimoune · J.-C. Lacherade · J.-B. Lascarroux · L. Legrain · Y. Macciotta $\cdot$ A. Mengus $\cdot$ S. Ory $\cdot$ S. Papin $\cdot$ S. Payen $\cdot$ F. Pereira $\cdot$ G. Piton $\cdot$ S. Rodriguez $\cdot$

T. Rodriguez $\cdot$ M. Simon $\cdot$ L. Laurent-Taluy $\cdot$ E. Toure $\cdot$ S. Turia $\cdot$ E. Villette $\cdot$ S. Bejaud $\cdot$ F. Merand $\cdot$ G. Muller $\cdot$ S. Hraiech · pour la commission d'épidémiologie et de recherche clinique de la SRLF (CERC SRLF Trial Group)
\end{abstract}

Reçu le 12 janvier 2018; accepté le 19 mai 2018

(C) SRLF et Lavoisier SAS 2018

A. Youssoufa $\cdot$ S. Hraiech $(\bowtie)$

Service de médecine intensive-réanimation, AP-HM, CHU Nord Marseille, F-13015 Marseille, France

e-mail : sami.hraiech@ap-hm.fr

G. Decormeille

Service de réanimation, $\mathrm{CHU}$ de Toulouse,

F-31059 Toulouse, France

P. Michel

Service de réanimation, centre hospitalier Pontoise,

F-95303 Pontoise, France

G. Jacq

Service de réanimation, centre hospitalier de Versailles,

F-78157 Versailles, France

F. Brouard

Service de réanimation, centre hospitalier de Périgueux,

F-61205 Périgueux, France

N. Aissaoui

Service de réanimation, HEGP, AP-HP Paris,

F-75015 Paris, France

S. Barbar

Service de réanimation, CHU de Nîmes, F-30029 Nîmes, France

N. Belaizi

Service de réanimation, CHU de Nice, F-06202 Nice, France

F. Boissier

Service de réanimation, CHU de Poitiers, ,

F-86021 Poitiers, France
C. Boulinguiez $\cdot$ J.-B. Lascarroux

Service de réanimation, CHU de Nantes, F-44093 Nantes, France

V. Chauvin $\cdot$ S. Papin

Service de réanimation, centre hospitalier de Belfort,

F-90000 Belfort, France

C. Corrolleur

Service de réanimation, centre hospitalier de Brest,

F-29200 Brest, France

S. Dame $\cdot$ Y. Macciotta $\cdot$ L. Laurent-Taluy

Service de réanimation, centre hospitalier de Chambéry,

F-73000 Chambéry, France

D. Da Silva $\cdot$ S. Turia

Service de réanimation, centre hospitalier de Saint-Denis,

F93205 Saint-Denis, France

J. Dauvergne

Service de réanimation, centre hospitalier de Nantes,

F-44093 Nantes, France

J. Domitien $\cdot$ A. Joosten $\cdot$ M. Simon

Service de réanimation, centre hospitalier de Charleroi,

Boulevard Zoé Drion, B-6000 Charleroi, Belgique

G. Fouquet · A. Kimoune

Service de réanimation, CHU de Nancy,

F-54000 Nancy, France

C. Garin

Service de réanimation, HEGP, AP-HP, Paris,

F-75015 Paris, France 
Résumé Objectifs : Bien que quotidiennement géré par les infirmier(ière)s diplômé(e)s d'État (IDE) de réanimation, le sevrage des catécholamines a rarement été étudié. L'hypothèse de notre étude était que l'augmentation du nombre d'interventions menées sur la vitesse de perfusion des catécholamines permettait de raccourcir la durée d'administration de celles-ci.

Patients et méthodes : Il s'agissait d'une étude prospective observationnelle dans 21 réanimations. Tous les patients traités par vasopresseurs pendant la période d'étude ont été inclus. Le nombre d'interventions effectuées par l'IDE en charge du patient sur la vitesse d'administration des catécholamines était noté toutes les quatre heures. La posologie de catécholamines en cours ainsi que la pression artérielle moyenne (PAM) du patient étaient également relevées. Les facteurs influençant la rapidité du sevrage des catécholamines étaient également analysés.

Résultats : Nous avons inclus 226 patients dont l'âge moyen était de $65 \pm 14$ ans. La durée moyenne du traitement par catécholamines était de 71,6 $( \pm 81)$ heures. Le nombre d'interventions IDE par quatre heures était de 1,9 $( \pm 2,8)$ intervention. La durée du traitement par catécholamines était significativement moindre lorsque le nombre d'interventions augmentait $(p=0,04)$. L'existence d'un protocole spécifique à la gestion des catécholamines raccourcissait leur durée de sevrage. Un IGS2 élevé, une PAM élevée

P. Gay $\cdot$ T. Rodriguez

Service de réanimation, CHU de Lyon, F-69003 Lyon, France

D. Grimaldi

Service de réanimation, hôpital Érasme, Bruxelles,

Route de Lennik 800, B-1070 Bruxelles, Belgique

O. Hamzaoui

Service de réanimation, CHU Antoine-Béclère, AP-HP,

F-92140 Clamart, France

J.-C. Lacherade

Service de réanimation, centre hospitalier de La-Roche-sur-Yon, F-85925 La-Roche-sur-Yon , France

L. Legrain

Service de réanimation, CHU Pitié-Salpêtrière, AP-HP, F-75013 Paris, France

A. Mengus

Service de réanimation, centre hospitalier de Mulhouse, F-68100 Mulhouse, France

\section{S. Ory}

Service de réanimation, CHU de Genève,

Rue Gabrielle-Perret-Gentil 4, CH-1205, Genève, Suisse

S. Payen

Service de réanimation, centre hospitalier de Boulogne-

Billancourt, F-95100 Boulogne-Billancourt, France ainsi que la période diurne étaient associés à une augmentation du nombre des interventions IDE.

Conclusion : Le nombre d'interventions par les IDEs menées sur la perfusion de catécholamines était inversement lié à la durée du traitement par catécholamines. Des études complémentaires permettront d'évaluer l'impact de ces interventions sur le pronostic des patients.

Mots clés Catécholamines · Sevrage · Infirmiers diplômés d'État · Réanimation

Abstract Objectives: Although daily managed by ICU nurses, weaning from vasopressors has rarely been studied. The aim of our study was to determine if the increase of number of interventions carried out by nurses on vasopressors infusion regimen reduces catecholamine's treatment duration. Patients and methods: We have conducted an observational prospective study in 21 ICUs. All patients treated with vasopressors (epinephrine and/or norepinephrine) during the study period were included. The number of interventions (vasopressors infusion regimen increase or decrease) carried out by the nurse in charge of the patient was noted every $4 \mathrm{~h}$ and divided by the total duration of administration. The current vasopressor dosage and the patient's mean arterial blood pressure (MAP) were also recorded. Factors influencing the rapidity of withdrawal from vasopressors were also analyzed.

F. Pereira

Service de réanimation, CHU Pitié-Salpêtrière,

AP-HP, F75013 Paris, France

G. Piton

Service de réanimation, CHU de Besançon,

F-25000 Besançon, France

S. Rodriguez

Service de réanimation, CHU Tenon, F-75020 Paris France

E. Toure

Service de réanimation, CHU de Colombes,

F-92701 Colombes France

E. Villette

Service de réanimation, HEGP, AP-HP, Paris, F-75015 Paris, France

S. Bejaud $\cdot$ F. Merand

Service de réanimation médicale, CHU de Poitiers,

F-86021 Poitiers, France

G. Muller

Service de réanimation, centre hospitalier d'Orléans,

F-45067 Orléans, France

pour la commission d'épidémiologie et de recherche clinique de la SRLF (CERC SRLF Trial Group) Maison de la Réanimation, 48 avenue Claude Vellefaux, F-75010 Paris, France 
Results: We have included 226 patients whose mean age was $65 \pm 14$ years. The mean vasopressors treatment duration was $71.6( \pm 81) \mathrm{h}$. The number of nurse interventions per $4 \mathrm{~h}$ was $1.9( \pm 2.8)$. The duration of administration of vasopressors was significantly lower when the number of interventions increased $(P=0.04)$. In a multivariable analysis, SAPSII (Simplified Acute Physiology Score 2) value, MAP, existence of a weaning protocol, and the day period were associated to an increased number of nurse interventions.

Conclusion: The number of nurse interventions related to vasopressors treatment regimen modification was inversely correlated to vasopressors treatment duration. Additional studies were warranted to assess the impact of these interventions on patients' prognosis.

Keywords Vasopressors $\cdot$ Weaning $\cdot$ Nurse $\cdot$ Intensive care unit

\section{Introduction}

L'instabilité hémodynamique des patients hospitalisés en réanimation nécessite fréquemment l'administration de catécholamines, d'inotropes ou de vasopressives [1]. Les amines vasopressives sont utilisées au cours des états de choc avec une composante vasoplégique. La manipulation de ces molécules a des conséquences importantes et rapides sur l'état hémodynamique des patients. Leur administration est gérée au quotidien par les infirmier(ière)s diplômé(e)s d'État (IDE) de réanimation sur prescription médicale. La plupart des études paramédicales ayant évalué la gestion des catécholamines en réanimation concernent les modalités pratiques et les conséquences du relais de ces traitements [2-4]. Pourtant, dans la plupart des réanimations, les IDEs gèrent également les augmentations et les baisses des posologies en fonction d'une valeur cible de pression artérielle (systolique, diastolique ou moyenne) pouvant différer selon les médecins ou les services. Par ailleurs, les modalités de baisse ou d'augmentation sont variables en fonction des services, mais également parfois à l'intérieur d'une même unité selon le prescripteur. Si l'utilisation d'un protocole permet probablement d'éviter les variations hémodynamiques délétères au cours du sevrage, l'impact sur la durée de traitement par vasopresseurs a rarement été étudié [5], et il n'existe aucune recommandation claire des sociétés savantes sur ce sujet. Il n'existe à l'heure actuelle aucune donnée permettant de savoir si le nombre et le rythme des modifications posologiques de vasopresseurs réalisées par l'IDE impactent leur durée d'administration. Plusieurs études ont pourtant montré qu'une administration prolongée de catécholamines était associée à une augmentation de la mortalité $[6,7]$.
L'objectif principal de cette étude était de tester l'hypothèse qu'une augmentation du nombre d'interventions effectuées par l'IDE sur la vitesse de perfusion des catécholamines permettait d'en raccourcir la durée de traitement.

Les objectifs secondaires étaient de décrire les facteurs influençant le sevrage de vasopresseurs effectué par les IDEs.

\section{Patients et méthodes}

Nous avons conduit une étude multicentrique, prospective, observationnelle au sein de réanimations médicales, chirurgicales et polyvalentes francophones en France, en Suisse et en Belgique. Cette étude a été conçue et menée par des IDEs exerçant en réanimation ayant participé à la « Journée d'initiation du personnel paramédical à la recherche clinique » de mars 2016, formation organisée par la commission d'épidémiologie et de recherche clinique de la SRLF. L'étude a été approuvée par le comité d'éthique de la SRLF (numéro 16-25).

Tous les patients adultes hospitalisés en réanimation nécessitant l'administration d'amines vasopressives depuis moins de 24 heures sur une voie veineuse centrale (VVC) étaient éligibles, à l'exclusion des patients chez qui les vasopresseurs étaient perfusés sur des cathéters à chambre implantable $(\mathrm{CCI})$ ou des cathéters veineux centraux à abord périphérique (PICC line).

Les patients présentant un des critères suivants étaient exclus :

- patient admis pour arrêt cardiaque ;

- patient traité par dobutamine seule ;

- patient ayant un cœur artificiel total ;

- patient en état de mort encéphalique ;

- femme enceinte ou allaitante ;

- patient sous tutelle/curatelle;

- patient moribond ou décédé moins de 24 heures après leur inclusion.

Les patients participaient à l'étude de l'introduction des vasopresseurs jusqu'à leur sevrage.

Une information orale était délivrée aux patients ou à leur personne de confiance avant l'inclusion dans l'étude, et l'absence d'opposition était recueillie.

Les données concernant les caractéristiques démographiques du patient ainsi que celles relatives à la VVC utilisée et à la prescription de catécholamines étaient recueillies à l'inclusion. Pendant la durée de participation à l'étude, le recueil de données portait sur le nombre d'interventions de l'IDE sur la posologie de vasopresseurs (augmentation ou diminution), la posologie des vasopresseurs en cours ainsi que la PAM du patient au même moment toutes les quatre heures.

L'IDE ayant en charge le patient inclus devait comptabiliser sur une feuille de recueil dédiée au protocole le nombre 
de fois où il (elle) modifiait la posologie des amines reçues en indiquant s'il augmentait ou diminuait la vitesse de la seringue électrique. Toutes les quatre heures étaient notés le nombre total d'interventions, la posologie de catécholamines en cours ainsi que la PAM du patient. Ces données étaient ensuite saisies dans un CRF électronique. La durée totale d'administration des vasopresseurs depuis leur introduction jusqu'au premier sevrage complet était reportée pour chaque patient.

Le critère de jugement principal était le nombre d'interventions de l'IDE sur les posologies de catécholamines rapporté au nombre de jours de traitement par catécholamines. Les critères de jugement secondaires étaient le lien entre le nombre d'interventions IDE par période de quatre heures et des facteurs organisationnels du service ou liés au patient.

\section{Analyse statistique}

Les données discrètes étaient décrites par leur fréquence exprimée en pourcentage avec un intervalle de confiance à $95 \%$ et ont été comparées par le test du $\mathrm{Chi}^{2}$ ou le test exact de Fisher lorsque cela s'avérait nécessaire. Les intervalles de confiance ont été calculés par transformation angulaire. Les données numériques étaient décrites par leur moyenne et l'écart-type. Les données continues ont été comparées par le test de Student après vérification de l'égalité des variances ou par le test de Mann-Whitney. Les analyses multivariées étaient réalisées en régression logistique. Une analyse factorielle a également été réalisée. Les statistiques ont été effectuées grâce au logiciel R $\mid c i t e\{R \log \}$ ( R Foundation for Statistical Computing - Austria 2015).

L'objectif principal était modélisé par une corrélation entre le nombre d'interventions IDE sur les catécholamines et la durée de traitement par ces dernières. Le test de Spearman a été utilisé pour cette évaluation. Un calcul de puissance $a$ été fait après transformation par arc tangente en prenant un risque alpha à $5 \%$, un risque bêta à $20 \%$ et un rhô à 0,3 (corrélation moyenne).

\section{Résultats}

\section{Caractéristiques de la population}

Nous avons inclus 226 patients dans 21 centres participants entre le $1^{\text {er }}$ juin et le 31 août 2016. L'âge moyen de la population était de $65 \pm 14$ ans avec une prédominance masculine (68\%). La mortalité des patients inclus au cours de la période d'étude était de $32 \%$. Les caractéristiques démographiques des patients à l'inclusion, l'indication de prescription des catécholamines ainsi que les détails de prescription, d'administration et de sevrage des catécholamines sont donnés dans le tableau 1.

\begin{tabular}{|c|c|}
\hline \multicolumn{2}{|l|}{ Caractéristiques des patients } \\
\hline Âge (années \pm écart-type) & $65 \pm 14$ \\
\hline Sexe féminin $n(\%)$ & $72(32)$ \\
\hline Score IGS2 (médiane [IQR]) & $50[46-53]$ \\
\hline \multicolumn{2}{|l|}{ Traitement $n(\%)$} \\
\hline Ventilation mécanique & $161(81)$ \\
\hline Dont ventilation non invasive & $35(17)$ \\
\hline Épuration extrarénale & $46(24)$ \\
\hline ECMO VV & $9(4,6)$ \\
\hline ECMO VA & $4(2,1)$ \\
\hline \multicolumn{2}{|l|}{ Indication des catécholamines $n(\%)$} \\
\hline Choc septique & $102(56)$ \\
\hline Choc cardiogénique & $34(19)$ \\
\hline Choc hémorragique & $20(12)$ \\
\hline Vasoplégie en lien avec la sédation & $35(19)$ \\
\hline Neuroprotection & $28(16)$ \\
\hline \multicolumn{2}{|l|}{ Site de la VVC $n(\%)$} \\
\hline Fémoral & $65(33)$ \\
\hline Jugulaire & $104(53)$ \\
\hline Sous-clavier & $28(14)$ \\
\hline \multicolumn{2}{|l|}{ Type de VVC $n(\%)$} \\
\hline Bi-lumière & $15(7,6)$ \\
\hline Tri-lumière & $164(83)$ \\
\hline Autres & $18(9,1)$ \\
\hline \multicolumn{2}{|l|}{ Voie des catécholamines $n(\%)$} \\
\hline Proximale & $186(95)$ \\
\hline Médiane & $5(2,6)$ \\
\hline Distale & $5(2,6)$ \\
\hline Voie exclusive aux catécholamines & $149(77)$ \\
\hline \multicolumn{2}{|l|}{ Prescription $n(\%)$} \\
\hline Objectif PAM & $160(80)$ \\
\hline Objectif systolique & $5(2,7)$ \\
\hline Objectif diastolique & $4(2,2)$ \\
\hline Quantité d'augmentation-baisse/changement & $15(7,6)$ \\
\hline Délai entre deux changements & $3(1,5)$ \\
\hline \multicolumn{2}{|c|}{$\begin{array}{l}\text { IQR : interquartile range; IGS2 : indice de gravité simplifié ; } \\
\text { ECMO VV : extracorporeal membrane oxygenation veinovei- } \\
\text { neuse ; VA : veinoartérielle ; VVC : voie veineuse centrale ; } \\
\text { PAM : pression artérielle moyenne }\end{array}$} \\
\hline
\end{tabular}

Lien entre le nombre d'interventions IDE et la durée d'administration des catécholamines

La durée moyenne de traitement par catécholamines était de $71,6( \pm 81)$ heures. Le nombre d'interventions infirmières par période de quatre heures était de $1,9( \pm 2,8)$ intervention.

Il existait une association significative entre le nombre d'interventions infirmières et la durée de traitement par 
catécholamines. Cette durée était significativement moindre lorsque le nombre d'interventions augmentait $(p=0,04)$ (Fig. 1).

\section{Facteurs influençant le nombre d'interventions}

Le nombre d'interventions IDE par périodes de quatre heures était significativement plus élevé lorsque la PAM était élevée (Fig. 2). De même, les interventions IDE étaient plus fréquentes lorsque les posologies de catécholamines utilisées étaient élevées (Fig. 3).

En analyse multivariée, l'existence d'un protocole spécifique à la gestion des catécholamines propre au service raccourcissait la durée d'administration des catécholamines. Un IGS2 élevé, une PAM élevée étaient également associés à une augmentation du nombre des interventions IDE par période de quatre heures. Enfin, les interventions IDE étaient plus nombreuses le jour que la nuit (Tableau 2).

En revanche, la présence d'une extracorporeal membrane oxygenation (ECMO), la ventilation non invasive (VNI), le site où était implantée la VVC n'étaient pas associés à une modification du nombre d'interventions par les IDEs.

Il n'existait pas de lien entre le nombre d'interventions IDE par quatre heures et la durée de séjour en réanimation.

\section{Discussion}

Cette étude montre que le nombre d'interventions par quatre heures menées par les IDEs sur la vitesse de perfusion des catécholamines est inversement lié à la durée du traitement par catécholamines. Elle montre également que les catécholamines sont plus rapidement sevrées dans les services où il existe un protocole précis permettant de guider les IDEs dans

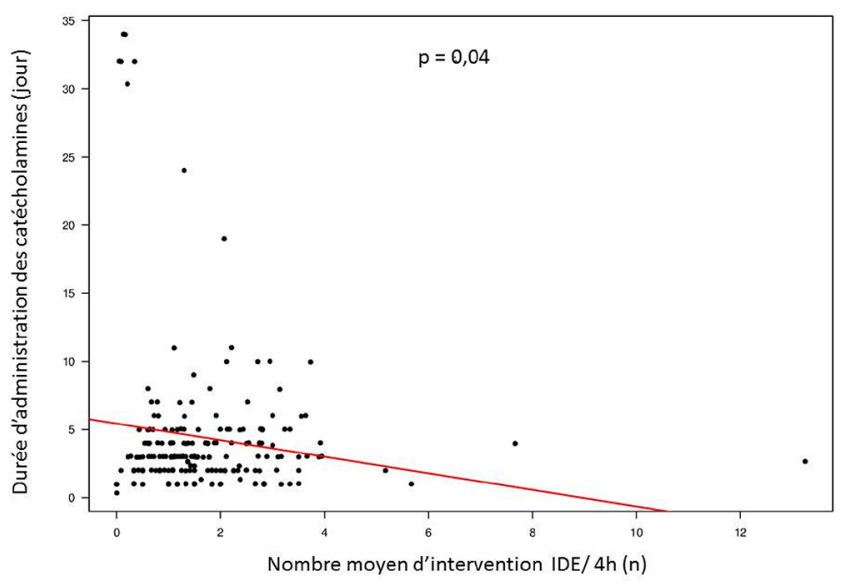

Fig. 1 Association entre le nombre d'interventions des IDEs par période de quatre heures sur la vitesse de perfusion des catécholamines et le nombre de jours de traitement par catécholamines $\mathrm{La}$ ligne rouge représente la droite de régression

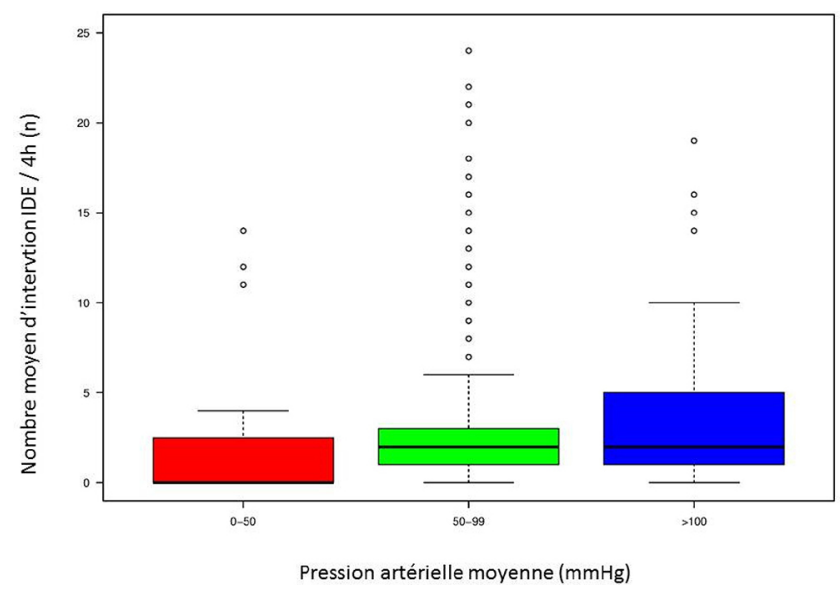

Fig. 2 Association entre le nombre d'interventions des IDEs par période de quatre heures sur la vitesse de perfusion des catécholamines et la pression artérielle moyenne des patients Les boîtes à moustache représentent les $25^{\mathrm{e}}$ et $75^{\mathrm{e}}$ percentiles, les barres représentent les $5^{\mathrm{e}}$ et $95^{\mathrm{e}}$ percentiles. La médiane est représentée par le trait horizontal PAM : pression artérielle moyenne ; IDE : infirmier(ière) diplômé(e) d'État

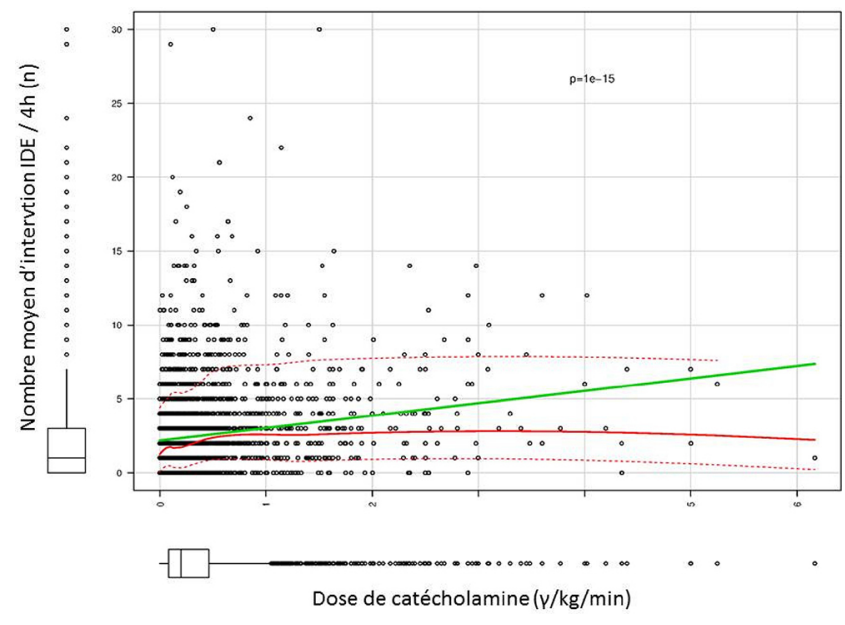

Fig. 3 Association entre le nombre d'interventions des IDEs par période de quatre heures sur la vitesse de perfusion des catécholamines et la pression artérielle moyenne des patients La ligne verte représente la droite de régression, la ligne rouge est la moyenne mobile avec son intervalle de confiance

le sevrage. Il apparaît également que des facteurs propres au patient et reflétant sa sévérité sont à l'origine d'un nombre plus important d'interventions infirmières sur la posologie de catécholamines.

Ce travail met en avant le fait que la durée d'administration n'est pas uniquement liée à la restauration d'une hémodynamique stable, mais que le facteur humain paramédical entre également en jeu. Des résultats comparables avaient été retrouvés en comparant un sevrage total de noradrénaline 
Tableau 2 Paramètres associés de façon significative avec le nombre d'interventions IDE par période de quatre heures en analyse uni- et multivariée

\begin{tabular}{|lllll|}
\hline Variables & \multicolumn{2}{c|}{ Analyse univariée } & & \multicolumn{2}{c|}{ Analyse multivariée } \\
\cline { 2 - 5 } & Valeur de $\boldsymbol{p}$ & Odds ratio & IC 95 \% & Valeur de $\boldsymbol{p}$ \\
\hline Existence d'un protocole de service & $<0,001$ & 1,03 & $(1,02-1,05)$ & $<0,001$ \\
Pression artérielle moyenne & $<0,001$ & 1,01 & $(1-1,02)$ & 0,003 \\
Période jour/nuit & $<0,001$ & 0,98 & $(0,97-0,98)$ & $<0,001$ \\
IGS2 & $<0,001$ & 1,01 & $(1-1,02)$ & 0,001 \\
\hline IGS2 : indice de gravité simplifié & & & & \\
\hline
\end{tabular}

laissé à l'appréciation des cliniciens à un sevrage réalisé via une boucle informatisée [8]. Dans cette étude, il existait une réduction importante de la durée de l'état de choc et de la posologie cumulée de noradrénaline dans le groupe interventionnel. Il est à noter qu'il n'existait pas de protocole de sevrage paramédical dans le groupe témoin. Cela revêt une importance particulière puisqu'il a été démontré qu'une durée longue de traitement par catécholamines était un facteur de mauvais pronostic chez les patients séjournant de façon prolongée en réanimation [6]. Dans ce travail, un traitement par inotropes ou vasopresseurs d'une durée supérieure ou égale à trois jours à $\mathrm{j} 30 \mathrm{ou}$ au-delà était un facteur prédictif de surmortalité hospitalière. Dans notre étude, la durée moyenne d'administration des catécholamines était inférieure à trois jours, mais n'était cependant pas calculée sur l'ensemble du séjour, les patients étant suivis jusqu'au sevrage total des catécholamines et ne pouvant être inclus qu'une seule fois dans l'étude. Nos résultats soulignent toutefois que cette durée d'administration des catécholamines, souvent considérée comme un marqueur de sévérité du patient, est également influencée par les interventions des IDEs. Ils mettent également en évidence que l'existence d'un protocole de service favorise un sevrage plus rapide des catécholamines par les IDEs probablement, car ils encadrent mieux l'application de la prescription médicale que lorsque celle-ci repose uniquement sur un objectif de pression artérielle.

Cette étude est une des premières à s'intéresser à la gestion des catécholamines par les IDEs. En effet, il a été démontré que des protocoles de sédation gérés par les IDEs selon un algorithme précis dans le but d'obtenir la plus faible dose efficace permettent de réduire la mortalité en réanimation, la durée de ventilation mécanique, de séjour en réanimation et à l'hôpital [9]. De même, des protocoles d'administration de l'insuline sont couramment gérés par les IDEs de réanimation et permettent un meilleur contrôle de la glycémie avec en particulier une diminution des hypoglycémies sans augmenter la charge de travail paramédicale [10]. Une étude récente s'est intéressée à la gestion paramédicale protocolisée de la curarisation au cours du syndrome de détresse respiratoire aiguë de l'adulte (SDRA) et montre qu'elle permet une réduction des posologies de cisatracurium utilisées [11]. Ces données ainsi que nos résultats plaident pour une protocolisation plus précise du sevrage des catécholamines par les IDEs. En effet, nos résultats montrent que la quantité de diminution ou d'augmentation de la vitesse de pousse-seringue électrique ou le délai entre deux modifications n'étaient que très rarement prescrits même lorsqu'il existait un protocole de sevrage dans le service. De plus, une accentuation de la formation et de la sensibilisation des équipes de nuit mériterait d'être faite puisque celles-ci ont tendance à moins sevrer les catécholamines alors que les objectifs de pression artérielle sont atteints. Cela est probablement dû à l'effectif médical restreint au cours de la période de permanence des soins.

Cette étude a cependant plusieurs limites. Tout d'abord, les patients n'étaient suivis que pendant la durée de leur participation à l'étude, c'est-à-dire jusqu'au sevrage des catécholamines. De ce fait, nous n'avons pu calculer le nombre de jours vivant sans catécholamines. Néanmoins, l'exclusion des patients décédés dans les 24 heures après leur inclusion nous permettait de limiter le biais lié à une durée d'administration courte de catécholamines en raison d'un décès précoce. De plus, s'il existe bien une association statistique entre le nombre d'interventions par quatre heures et la durée d'administration des catécholamines, il est difficile de conclure à un lien de causalité entre ces deux éléments. Enfin, nous n'avons pas montré que l'augmentation du nombre d'interventions IDE par quatre heures s'accompagnait d'une réduction de la durée de séjour. Un facteur confondant explicatif est sans doute que les patients avec les plus fortes posologies de catécholamines avaient significativement plus d'interventions IDE par quatre heures, cela étant probablement un marqueur de gravité, corrélé à une durée de séjour plus longue. Il faut noter que l'étude de Merouani et al. [8] ne retrouvait pas d'impact de la réduction de la durée d'administration de noradrénaline sur le pronostic des patients (en particulier le nombre de jours vivant en dehors de la réanimation). 


\section{Conclusion}

Cette étude paramédicale montre que le nombre d'interventions des IDEs était inversement lié à la durée d'administration des catécholamines. Cela suggère que les IDEs ont un rôle crucial pour raccourcir la durée de traitement par vasopresseurs. Des protocoles de sevrage précis pourraient permettre de raccourcir encore cette durée. Cette étude ouvre la voie à de futurs travaux, en particulier pour mettre en évidence un impact de ces interventions IDE sur le pronostic des patients de réanimation, en particulier sur leur durée de séjour.

Liens d'intérêts : les auteurs déclarent ne pas avoir de lien d'intérêt.

\section{Références}

1. Kato R, Pinsky MR, (2015) Personalizing blood pressure management in septic shock. Ann Intensive Care 5: 41

2. Argaud L, Cour M, Martin O, Saint-Denis M, Ferry T, Goyatton A, Robert D, (2007) Changeovers of vasoactive drug infusion pumps: impact of a quality improvement program. Crit Care 11: R133

3. Morrice A, Jackson E, Farnell S, (2004) Practical considerations in the administration of intravenous vasoactive drugs in the criti- cal care setting. Part II - how safe is our practice? Intensive Crit Care Nurs 20: 183-9

4. Greau E, Lascarrou JB, Le Thuaut A, Maquigneau N, Alcourt Y, Coutolleau A, Rousseau C, Erragne V, Reignier J, (2015) Automatic versus manual changeovers of norepinephrine infusion pumps in critically ill adults: a prospective controlled study. Ann Intensive Care 5: 40

5. Arellano DL, Hanneman SK, (2014) Vasopressor weaning in patients with septic shock. Crit Care Nurs Clin North Am 26: $413-25$

6. Friedrich JO, Wilson G, Chant C, (2006) Long-term outcomes and clinical predictors of hospital mortality in very long stay intensive care unit patients: a cohort study. Crit Care 10: R59

7. Goncalves JA, Hydo LJ, Barie PS, (1998) Factors influencing outcome of prolonged norepinephrine therapy for shock in critical surgical illness. Shock 10: 231-6

8. Merouani M, Guignard B, Vincent F, Borron SW, Karoubi P, Fosse JP, Cohen Y, Clec'h C, Vicaut E, Marbeuf-Gueye C, Lapostolle F, Adnet F, (2008) Norepinephrine weaning in septic shock patients by closed loop control based on fuzzy logic. Crit Care 12: R155

9. De Jonghe B, Bastuji-Garin S, Fangio P, Lacherade JC, Jabot J, Appéré-De-Vecchi C, Rocha N, Outin H, (2005) Sedation algorithm in critically ill patients without acute brain injury. Crit Care Med 33: 120-7

10. Compton F, Ahlborn R, Weidehoff T, (2017) Nurse-directed blood glucose management in a medical intensive care unit. Crit Care Nurse 37: 30-40

11. Hraiech S, Forel JM, Guervilly C, Rambaud R, Lehingue S, Adda M, Sylla P, Valera S, Carvelli J, Gainnier M, Papazian L, Bourenne J, (2017) How to reduce cisatracurium consumption in ARDS patients: the TOF-ARDS study. Ann Intensive Care 7: 79 